\title{
Redefining the modular organization of the core Mediator complex
}

Xuejuan Wang ${ }^{1}$, Qianqian Sun ${ }^{1}$, Zhenrui Ding ${ }^{1}$, Jinhua Ji ${ }^{1}$, Jianye Wang ${ }^{1}$, Xiao Kong ${ }^{1}$, Jianghong Yang ${ }^{1}$, Gang Cai ${ }^{1,2,3}$

${ }^{I}$ School of Life Sciences, University of Science and Technology of China, ${ }^{2}$ Hefei National Laboratory for Physical Sciences at the Microscale, Center for Integrative Imaging, ${ }^{3}$ Center for Biomedical Engineering, University of Science and Technology of China, 443 Huang-Shan Road, Hefei, Anhui 230027, China

The Mediator complex plays an essential role in the regulation of eukaryotic transcription. The Saccharomyces cerevisiae core Mediator comprises 21 subunits, which are organized into Head, Middle and Tail modules. Previously, the Head module was assigned to a distinct dense domain at the base, and the Middle and Tail modules were identified to form a tight structure above the Head module, which apparently contradicted findings from many biochemical and functional studies. Here, we compared the structures of the core Mediator and its subcomplexes, especially the first 3D structure of the Head + Middle modules, which permitted an unambiguous assignment of the three modules. Furthermore, nanogold labeling pinpointing four Mediator subunits from different modules conclusively validated the modular assignment, in which the Head and Middle modules fold back on one another and form the upper portion of the core Mediator, while the Tail module forms a distinct dense domain at the base. The new modular model of the core Mediator has reconciled the previous inconsistencies between the structurally and functionally defined Mediator modules. Collectively, these analyses completely redefine the modular organization of the core Mediator, which allow us to integrate the structural and functional information into a coherent mechanism for the Mediator's modularity and regulation in transcription initiation.

Keywords: Mediator; modular organization; nanogold labeling; Head + Middle modules

Cell Research (2014) 24:796-808. doi:10.1038/cr.2014.64; published online 9 May 2014

\section{Introduction}

Eukaryotic transcriptional regulation is largely focused on the initiation process, which involves recruitment of RNA polymerase II (RNAPII) and general transcription factors (TFIIA, TFIIB, TFIID, TFIIE, TFIIF, and TFIIH) to a promoter, followed by the assembly of the preinitiation complex (PIC) [1]. Both basal and activated transcriptions are critically dependent on the Mediator complex [2-5], which is preeminently responsible for PIC assembly in vivo and for conveying regulatory signals from DNA-binding transcription factors to PIC. Although Mediator plays an essential and critical role in the

Correspondence: Gang Cai

Tel/Fax: 8655163603802

E-mail: gcai@ustc.edu.cn

Received 22 October 2013; revised 13 February 2014; accepted 13 March 2014; published online 9 May 2014 regulation of transcription in all eukaryotes, the detailed architecture and molecular mechanisms of the complex remain largely elusive.

Based on extensive biochemical and genetic studies, the core Mediator subunits have been suggested to be organized into three functional modules, Head, Middle and Tail, and the modular architecture and subunit composition are conserved from yeast to human [6]. Electron microscopy (EM) has been used to obtain structural information about Mediator from the budding yeast $S$. cerevisiae [7-9], the fission yeast $S$. pombe [10], and human $[11,12]$. The low abundance, heterogeneity and intrinsic flexibility of Mediator have prevented the highresolution structural determination of the entire complex. To date, the best-resolved Mediator structure was from $S$. cerevisiae using cryo-EM $(\sim 28 \AA)$; however, the model bias with regard to particle selection and projectionmatching refinement were not alleviated [8]. Although the EM structures of the Mediator complex from several 
species have been determined, none of the subunits has yet been clearly localized in the complex by EM.

Due to the complexity, flexibility and modularity of the core Mediator, many basic questions, such as the boundaries of the three modules, how they are organized, and how they interact with each other, remain unresolved. Despite the breakthroughs in the determination of the atomic structure of the Head modules from $S$. cerevisiae and $S$. pombe [13-15], the assignment of the Head module in the core Mediator remains vague [16]. Previous EM studies suggested that the Head module corresponded to a distinct dense domain at the base, and the Middle/Arm and Tail modules folded back onto each other to form a tight structure [7, 17]. Unfortunately, the segmentation was based solely on a comparison of lowresolution EM reconstructions of the core Mediator in RNAPII-associated and free forms [7, 17], which was ambiguous and apparently contradicted the information acquired from the following biochemical and functional studies: (1) the Head and Middle modules were reported to form a functional core of the Mediator [18, $19]$, whereas the Head module is largely separated from the Middle module in the previous EM model; (2) the theoretical molecular weight (MW) suggests that the Tail module should be the largest component and the Head module should be the smallest; however, the calculated MWs of the three segmented modules based on the previous EM model are roughly the same [8]; (3) the extensive interaction of the C-terminal domain (CTD) of RNAPII with the Head module has been firmly established $[13,14]$; however, a recent EM study reported that CTD mainly binds to the Middle module of the Mediator [20]; (4) the RNAPII was typically visualized as binding to Mediator through the Tail and Middle modules in previous EM studies $[8,20]$, although the Head module is responsible for binding RNAPII. These contradictory observations suggest that the previous understanding of the modular organization of the core Mediator is most likely confounded, posing a significant impediment to understanding the detailed architecture and molecular mechanisms of Mediator.

To understand how the core Mediator carries out multiple essential functions, it is critical to characterize the modular architecture, and to understand how the modules are organized and how they interact with each other to enable regulation of transcription. Here, we compared the EM structures of the core Mediator and its subcomplex, especially the first 3D structure of the Head + Middle modules, performed nanogold labeling of multiple subunits from different modules, and proposed a new modular organization of Mediator, which resolves many inconsistencies of the previous model with the biochemi- cal and functional studies. The redefined model allowed us to integrate the structural and functional information into a coherent mechanism for Mediator modularity and regulation in transcription initiation.

\section{Results}

Improved biochemical preparation of the core Mediator complex

The $S$. cerevisiae core Mediator complex comprises 21 subunits, which are organized into three functional and structural modules, including Head, Middle, and Tail (Figure 1A) [21]. It is extremely difficult to prepare homogeneous Mediator particles that are suitable for structural analyses. Previously, we established a purification procedure involving ammonium sulfate precipitation to enrich the Mediator-containing fraction and two steps of affinity chromatography using IgG and Ni-NTA columns [8]. To further remove any minor contamination, an ion exchange Mono Q column was employed (Supplementary information, Figure S1). The improved procedure yielded the nearly stoichiometric 21-subunit core Mediator complex, which was uniform in composition based on SDS-PAGE analysis (Figure 1B). The homogeneity and concentration of the core Mediator preparation were substantially improved using the new purification method, and we obtained a highly homogeneous Mediator preparation at a concentration of over $1 \mathrm{mg} / \mathrm{ml}$. The particles observed by EM appeared well-preserved and were similar in size and overall shape (Figure 1C).

Classification of structural heterogeneity of the core Mediator complex

Due to the substantial flexibility and extended shape of the core Mediator structure, the cryo images showed a very low signal-to-noise ratio and could not be stably aligned, leading to a considerable problem of model bias in the previous cryo reconstruction of the Mediator complex [8]. EM of heterogeneous particles preserved in negative staining produces much higher signal-to-noise ratio than unstained cryo-EM. On the other hand, while molecules prepared by vitrification usually adopt random orientations in the amorphous ice layer, negative staining tends to induce preferred orientations of the molecules on the carbon support film. By taking advantage of the preferred orientation induced by negative staining, image alignment and classification could be used to distinguish differences in the molecular conformation without having to simultaneously address differences in orientation. Therefore, we performed negative staining EM to characterize the structural variability of the core Mediator, and used the random conical tilt method to obtain 3D 


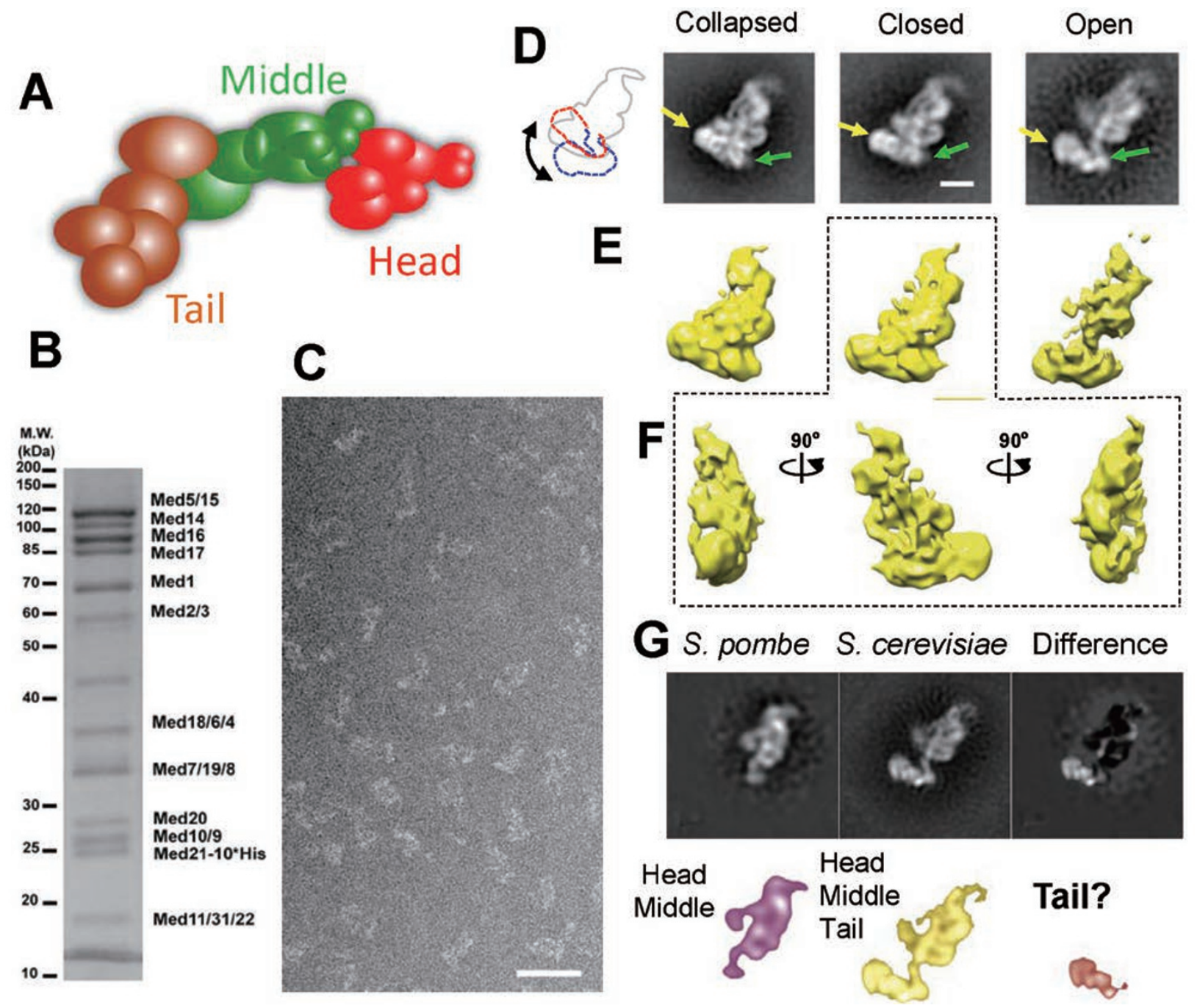

Figure 1 Structure of the core Mediator complex. (A) Schematic view of subunit and modular organization of the core Mediator from S. cerevisiae. (B) SDS-PAGE analysis of the native core Mediator purified from S. cerevisiae. The purifications were separated by SDS-PAGE, followed by Ruby staining. (C) A typical micrograph of the well-preserved particles under negative staining. Scale bar, $50 \mathrm{~nm}$. (D) Two-dimensional (2D) EM analysis of the core Mediator. Three different conformations were identified through reference-free alignment and classification of EM images, showing variability in the position of a dense domain at the base. Scale bar, $100 \AA$. (E) Three-dimensional (3D) reconstructions of the core Mediator in three conformations. Scale bar, $100 \AA$. (F) Different views of the 3D reconstructions of the core Mediator in closed conformation. (G) Comparison of the 2D structures of the core Mediator complex from S. pombe and S. cerevisiae and difference mapping establishes that the dense domain at the base of $S$. cerevisiae Mediator most likely corresponds to the Tail module, which is absent in S. pombe Mediator. Contour plots calculated from 2D structures of the core Mediators from S. pombe and S. cerevisiae are color-coded to highlight structural conservation and the approximate boundary of the Tail module.

reconstructions [22].

Three different conformations of the core Mediator were identified through the reference-free alignment and classification of EM images. The Mediator particles were nearly evenly distributed among the three different conformations (named collapsed, closed and open) (Figure 1D). The upper portion of the structure was largely stable, as indicated by reproducible structural details across different class averages. Further classification suggests that the structural variability among these three conformations was predominantly due to changes in the position of the dense domain at the bottom, which could be either separated from or abutted to the upper portion. The base domain pivoted on a central connection to the upper portion, which was stretched in the open conformation and condensed in the collapsed and closed conforma- 
tions. Moreover, the base domain appeared to undergo limited rotational rearrangement.

$3 D$ reconstructions of the core Mediator in three conformational states

Information from the images of tilted particles was used to obtain $3 \mathrm{D}$ reconstructions of the the complex in three different conformations (Figure 1E). It is noteworthy that these represent the first Mediator EM reconstructions in different conformational states. In contrast, the previously published EM structures were just the averaging of different conformations of the Mediator. By computationally sorting out different conformational states and reconstructing the structures from well-defined populations, the resulting 3D structures are considerably more intricate and provide new information for the Mediator structural organization, although the nominal resolution of the 3D reconstructions was not higher (Supplementary information, Figure S2) than that in previous studies. In particular, the structure of the upper portion of the Mediator, which was largely featureless in previous reconstructions, appeared much more complicated here, with several interconnected segments (Figure 1F).

Structural comparison of the core Mediator complexes from $S$. cerevisiae and $S$. pombe

Interestingly, the $S$. pombe Mediator contains only the Head and Middle modules [10]. According to comparisons of the crystal structures, the Head modules from $S$. cerevisiae and $S$. pombe are well-conserved despite the structured regions showing only $15 \%$ sequence identity [14]. To reinforce this observation, we determined the 2D structure of $S$. pombe Mediator; consistently, the structure of the Mediator from $S$. pombe closely resembled that of $S$. cerevisiae Mediator, especially the upper portion of the $S$. cerevisiae structure (Figure $1 \mathrm{G}$ ). The density of the movable dense domain at the base was entirely absent in the S. pombe structure (Figure 1G). Structural differences in Mediator between the two yeast species were further validated by difference mapping, a technique in which EM maps of a complex and a related stable subcomplex are compared to determine subunit localization. The $2 \mathrm{D}$ class average of the $S$. pombe Mediator matches that of the upper portion of $S$. cerevisiae Mediator in the open conformation, and the difference mapping conclusively identified the movable dense domain at the base as the structural difference. These observations reinforce the conservation of the Mediator structure despite the rather low sequence conservation of Mediator subunits [8, 14], and suggest that the distinct dense domain at the base most likely represents the Tail module that is absent in the $S$. pombe Mediator [10], instead of the Head module as previously indicated $[7,17]$.

\section{Structure of the Head + Middle modules}

To verify whether the dense domain at the base corresponds to the Tail module, we characterized the Head + Middle modules assemblage by taking advantage of a Med16 $\Delta$ mutant in which the Tail module dissociates $[23,24]$, and then compared the structure with that of the core Mediator. We purified the native Head + Middle modules from the Med16 $\Delta$ yeast strain by affinity tagging of Med21, a subunit of the Middle module (Figure 2A). Unexpectedly, we found that Med19 was stably copurified with the Middle module, which was previously identified as a component of the Head module [21] and was, however, absent from the endogenous purification of the Head module [13, 25]. Moreover, Med14, formerly assigned to the Tail module [21], was also stably co-purified with the Middle module (Figure 2B). These findings of Med19 and Med14 co-purification with the Middle module led to an updated map of the Mediator subunit and modular organization (Supplementary information, Figure S1) through integration with the published data [21].

The quality of the purification of Head + Middle modules was assessed by a direct examination of the homogeneity and integrity of the particles by EM. The particles were found to be highly pure and homogenous, and appeared well-preserved and similar in size and overall shape under negative staining (Figure 2C and Supplementary information, Figure S3). Upon adsorption to amorphous carbon support films, the Head + Middle particles also showed a strongly preferred orientation. The reference-free alignment and classification of the EM images of the Head + Middle modules generated several 2D averages corresponding to different conformations (Figure 2D and Supplementary information, Figure S3). In particular, an extended hammer-shaped domain at the left was variably connected to the stable right portion of the structure (Figure 2D). Information from images of tilted particles was used to obtain $3 \mathrm{D}$ reconstructions (Figure 2E), and the structural variability evident in the $2 \mathrm{D}$ averages was also clearly reflected in the 3D structures (Supplementary information, Figure S4). Because the protein concentration of the current preparation of Head + Middle modules was still very low $(<0.05 \mathrm{mg} / \mathrm{ml})$, we could not perform a cryo-EM reconstruction at the time.

\section{Boundary of the Tail module}

To obtain direct evidence for the localization of the Tail module, we compared the structure of the Head + Middle modules obtained from the Med16 $\Delta$ strain with that of the core Mediator. The structure of the Head + 


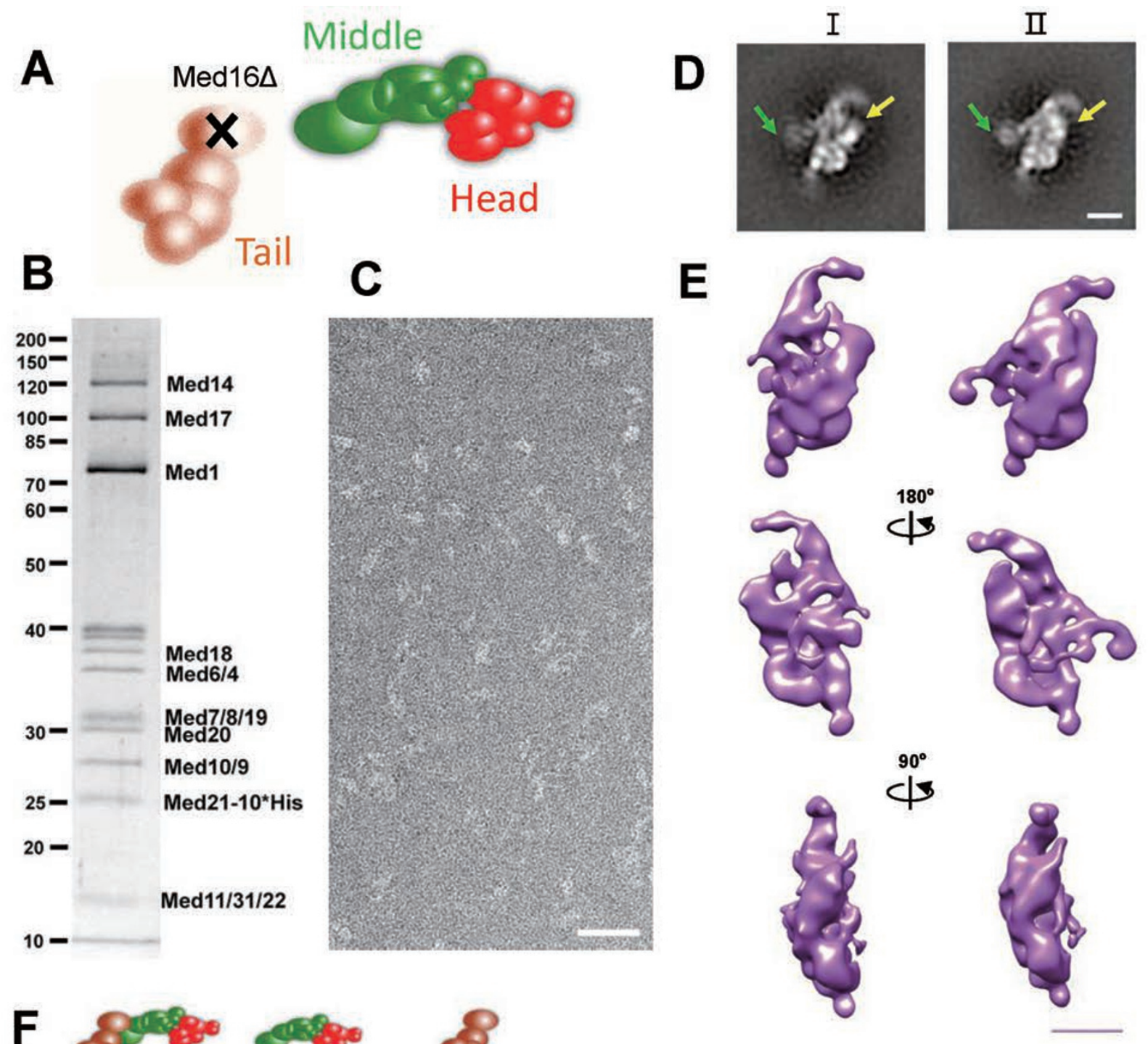

F

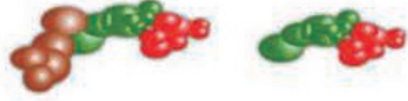

H
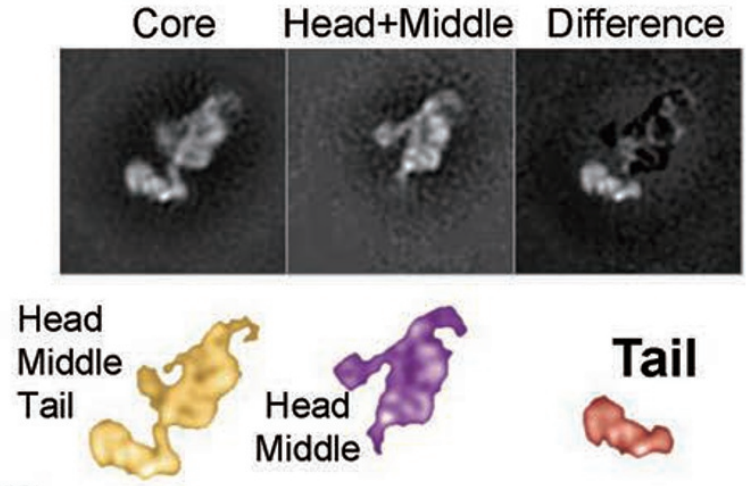

Tail

G
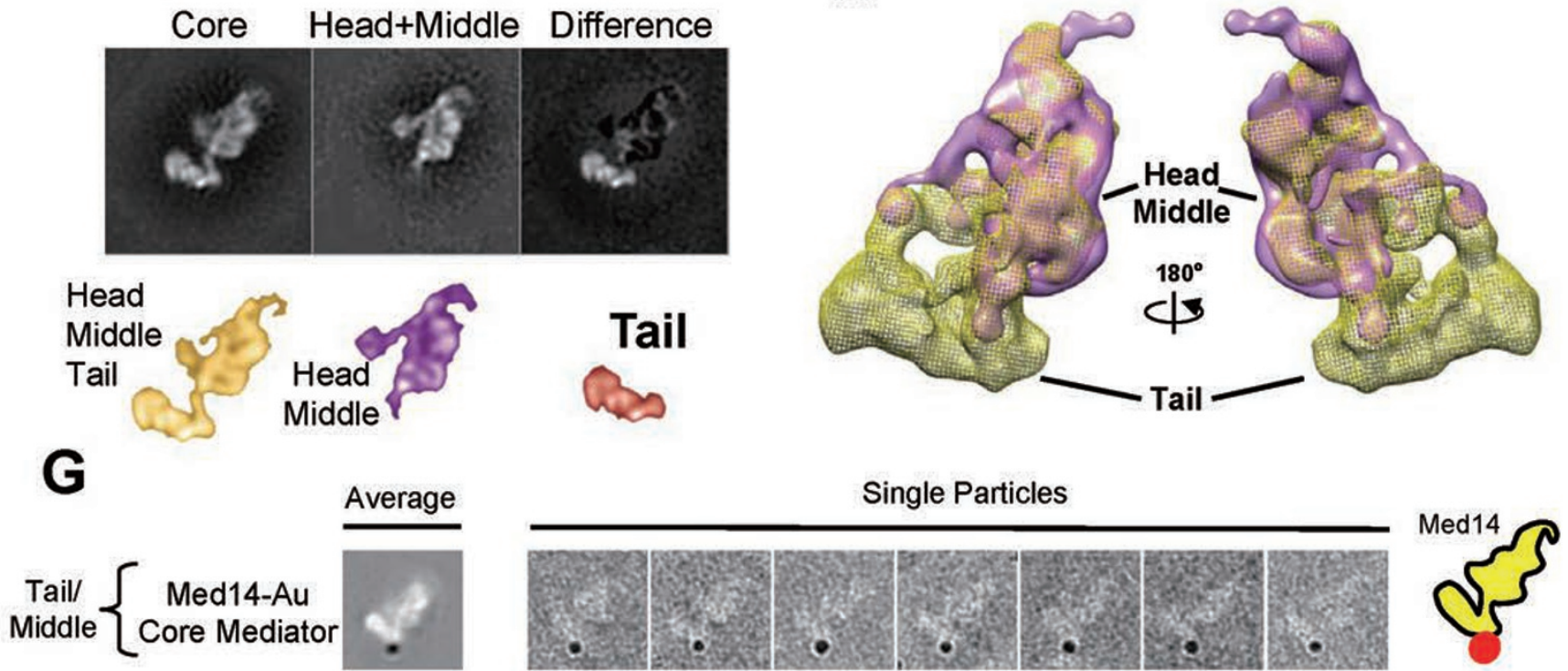
Middle modules matches that of the upper portion of the core Mediator in the open conformation, and difference mapping firmly identified the movable dense region at the base as the Tail module (Figure 2F). To further validate the localization of the Tail module, an oligohistidine tag was engineered onto the $\mathrm{C}$ terminus of Med14, which is a connector of the Middle and Tail module [21, 23]. After incubation the core Mediator particles with NiNTA nanogold probes, the class averages clearly showed a small, well-defined area of high density, representing a gold cluster (Figure 2G). The labeling specificity of the nanogold was confirmed by the absence of the dense spot in the single-particle images obtained from control preparations without the oligohistidine tag at the $\mathrm{C}$ terminus of Med14 (data not shown). The gold clusters clearly pinpointed that the $\mathrm{C}$ terminus of Med14 was located at the tip of the upper portion of the structure and inserted into the distinct dense domain at the base, which corresponded to the Tail module. This observation is consistent with the structural role of Med14 as a connector of the Middle and Tail modules [21, 23].

The localization of the Tail module was further validated by fitting the 3D EM structure of the Head + Middle modules into that of the core Mediator, which reveals that the Head + Middle modules correspond in size and shape to the upper portion of the core Mediator (Figure $2 \mathrm{H}$ ), while the dense region at the base corresponds to the Tail module, which accounts for more than $40 \%$ of the total density of the core Mediator. The peripheral position of the Tail module is consistent with its independent ability to interact with nucleosomes $[26,27]$ and transcription factors $[2,24]$, and its mobility must be at least partially related to the structural flexibility of the Med14 subunit, which is a connector of the Middle and Tail modules [21, 23]. Collectively, structural comparison of the core Mediator and the Head + Middle modules, combined with the nanogold labeling of the Med14 subunit of the Tail module, defines the boundary of the Tail module.

\section{Boundaries of the Head and Middle modules}

Further information about the boundaries of the Head and Middle modules was derived from a more detailed analysis of the structure of the Head + Middle modules. Comparison of the 2D and 3D structures of the Head + Middle modules and those of the Head module [28] indicates that the Head module matches the right-side portion of the Head + Middle modules in size and shape (Figure 3A). Moreover, difference mapping conclusively identified the right-side dense region of the Head + Middle modules as the Head module and the left-side elongated region as the Middle module (Figure 3A). Consistently, the crystal structure of the Head module (PDBID: 4GWP) [13] could be unambiguously fitted into the right portion of the Head + Middle modules, with the size and shape closely matched (Figure 3B), substantiating the localization of the Head module. Thus, we were able to calculate the first 3D structure of the Middle module by subtracting the Head module density from the structure of the Head + Middle modules (Figure 3B).

We further validated the localization of the Head and Middle modules by nanogold labeling of several Mediator subunits that belong to the two modules. An oligohistidine tag was engineered onto the $\mathrm{C}$ terminus of Med17 (Head module), Med22 (Head module) and Med21 (Middle module). After incubation with nanogold probes, the single-particle images and class averages of the core Mediator particles (or Head + Middle modules) clearly showed a dense spot, which corresponded to a gold cluster (Figure 3C). The positions of the nanogold clusters correctly reflect the localization of the corresponding tagged subunits in the complex. The gold clusters clearly pinpointed the C-termini of the Med17 and Med22 subunits at roughly the same position on the upper-right

Figure 2 Structure of the Head + Middle modules and boundary of the Tail module. (A) Schematic view of obtaining the Tailless core Mediator by deletion of the med16 gene. (B) SDS-PAGE analysis of the native Head + Middle modules. (C) A typical micrograph of the Head + Middle particles preserved under negative staining. Scale bar, $50 \mathrm{~nm}$. (D) Representative 2D structures of the Head + Middle modules. The yellow and green arrows indicate the regions showing high degree of structural flexibility. Scale bar, $100 \AA$. (E) Different views of the 3D reconstructions of the Head + Middle modules in two different conformations. Scale bar, $100 \AA$. (F) Comparison of the structures of the core Mediator and the Head + Middle modules and difference mapping establishes that the dense domain at the base of the core Mediator corresponds to the Tail module. Contour plots calculated from 2D structures are color-coded to highlight the modular correspondence and the boundary of the Tail module. (G) Localization of the $\mathrm{C}$ terminus of the Med14 subunit revealed by Ni-NTA-Nanogold labeling. Single particles of nanogold-labeled core Mediator complex (middle), a corresponding class average (left), and a diagram representing the localization of the Med14 C terminus (right) are shown. (H) Fitting of the structure of the Head + Middle modules (semitransparent purple surface) into the core Mediator structure (semitransparent yellow mesh). Comparison of the structures of the core Mediator and the Head + Middle modules combined with the nanogold labeling of the Med14 subunit firmly establishes the boundary of the Tail module. 
portion of the core Mediator, which directly validates the assignment of the Head module. This observation is consistent with the proximal localizations of the C-termini of these two subunits based on the crystal structure of the
Head module (PDBID: 4GWP) [13]. The C terminus of Med21 in the averages of the Head + Middle modules and the core Mediator particles was consistently pinpointed at the upper-left region of the structures (Figure

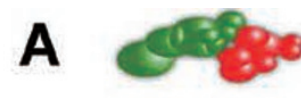

\section{(1)}

3D

Head+Middle

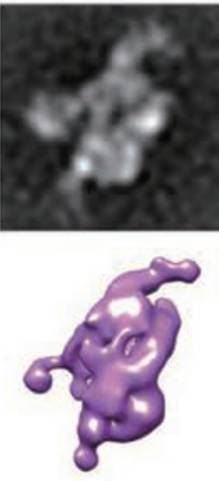

C

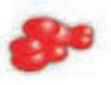

Head
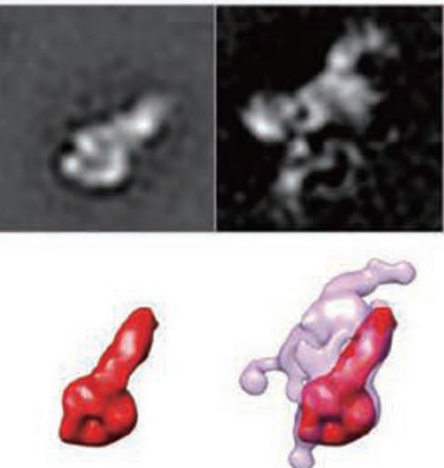

Averages

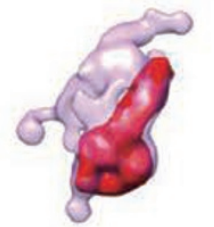

B

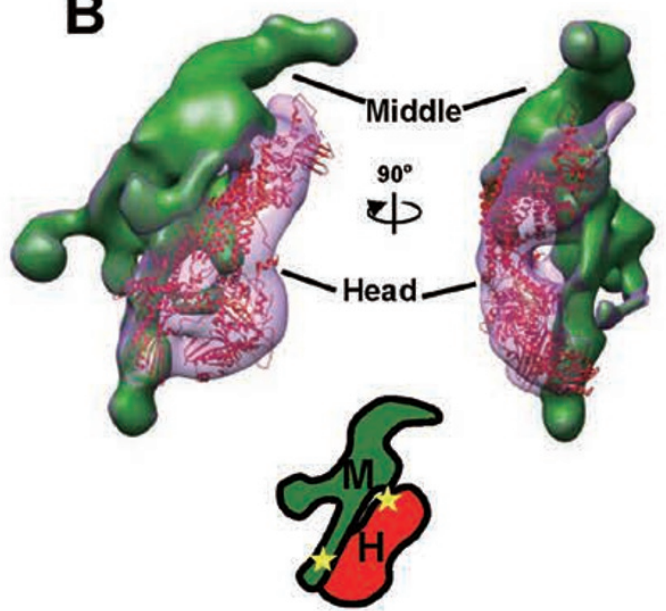

Single Particles
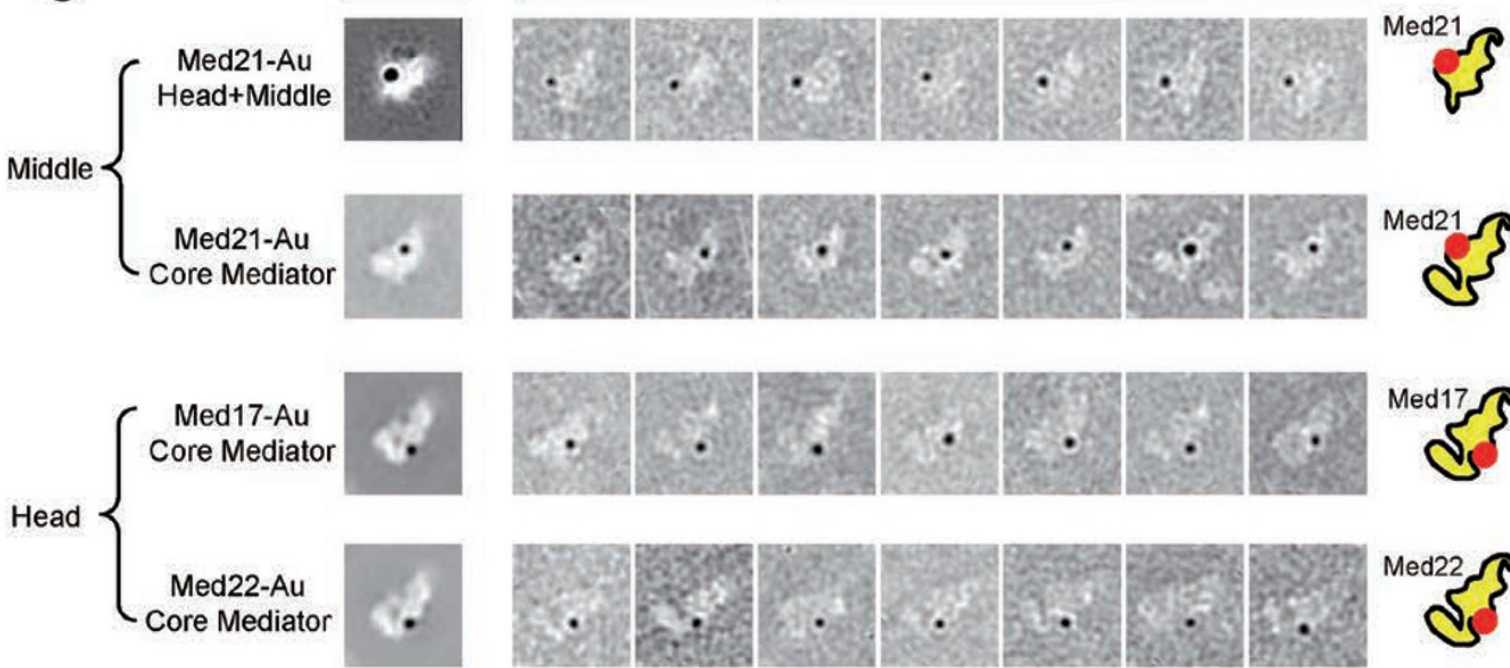

D

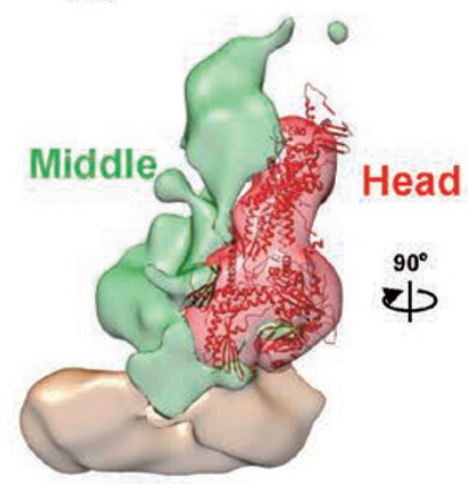

Tail
E Mediator+RNAPII

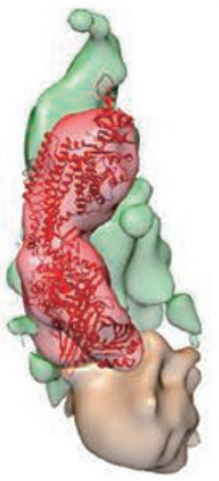

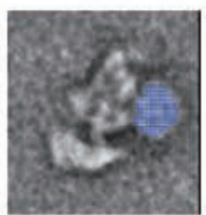

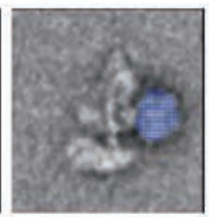

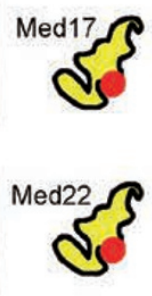
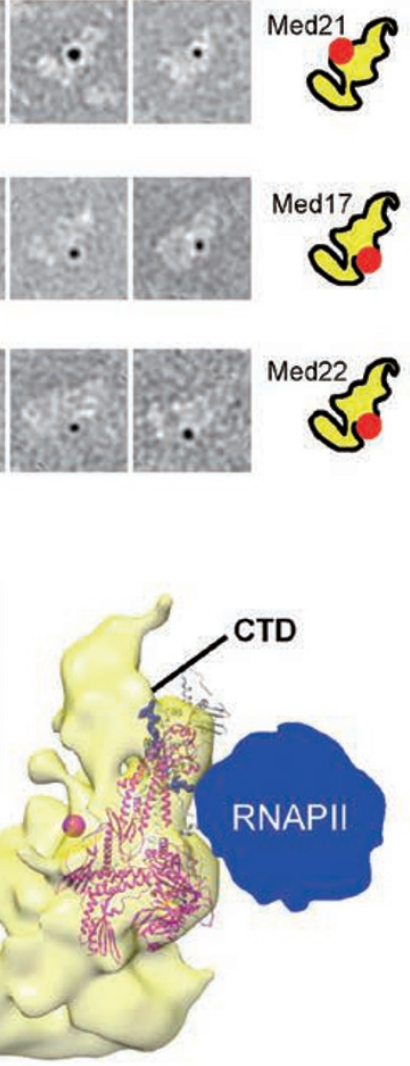
Figure 3 Boundaries of the Head and Middle modules. (A) Comparison of the structures of the Head + Middle modules and the Head module [28], combined with difference mapping, establishes that the Middle module corresponds to the extended domain at the left and the Head module forms the right portion of the structure. (B) Two views of the 3D reconstructions of the Head + Middle modules unambiguously fitted into the crystal structural model of the Head module (PDBID: 4GWP) [13]. The additional density related to the Middle module is represented as a green solid surface. A diagram (inset) revealing the boundary and connectors (yellow stars) between the Head and Middle modules is shown. (C) Localization of Mediator subunits from the Middle and Head modules revealed by using Ni-NTA nanogold probes against the His10 tag incorporated into the C-termini of Med21 (Middle), Med17 (Head) and Med22 (Head). Single particles of the nanogold-labeled core Mediator (middle), corresponding class averages (left) and diagrams representing the localization of the $\mathrm{C}$ terminus of each subunit (right) are shown. (D) Fitting the crystal structural model of the Head module (PDBID: 4GWP) [13] into the core Mediator structure (semitransparent colors according to the module segmentation). Comparison of the structures of the core Mediator and module assemblages, combined with the nanogold labeling of several subunits of different modules, completely redefines the modular organization of the core Mediator. (E) Representative class averages of the core Mediator interacting with RNAPII (left) and a figure representing the interaction modes of Mediator with RNAPII (right). The CTD of RNAPII deduced from the crystal structure (PDBID: 4GWQ) [13] is highlighted as the blue sphere. The X-ray structures of the four Srb subunits [13] (Med17/Srb4, Med18/Srb5, Med20/Srb2, and Med22/Srb6) in the Head module are shown in purple ribbon, and the only Srb subunit in the Middle module (Med21/Srb7) is represented by a purple ball.

3C). Therefore, nanogold labeling of the different Mediator subunits conclusively validated the localizations of the Head and Middle modules. The Head module forms the upper-right portion of the core Mediator, which resembles a closed wrench, while the Middle module corresponds to the upper-left portion, which is very elongated and extended.

The Head module was largely stable in conformation, as indicated by reproducible structural details across different class averages, and was readily separated from the Middle module in some conformations (Supplementary information, Figure S5). Interestingly, 3D reconstructions of the Head + Middle modules at a lower density threshold only revealed the structural features of the Head module, indicating that the Head module acts as the scaffold for Head + Middle assembly (Supplementary information, Figure S3). Detailed structural analysis demonstrates that the Middle module could be flexibly connected to the Head module through one or two connectors (Figure 3B), of which one is stable and the other is dynamic. The stable connector is centered on the fixed jaw region of the Head module. This connection perhaps represents the interaction between the Med17 and Med19 subunits [21], which could explain why deletion of Med19 leads to the dissociation of only the Middle module, whereas the Head and Tail modules remain tightly bound [25]. The dynamic connecting point around the shoulder region of the Head module, corresponding to Med6, most likely represented the interaction between Med6 and Med21 [14, 29], which was supported by the localization of Med21 (Figure 3C). Interestingly, the two identified inter-modular interaction points correspond to the arm and nose regions of the Head module, respectively, which have been suggested to bind the Middle module [14]. Consistent with this observation, the srb4-
138 mutant, with an apparent destabilization of both regions, exhibits a Mediator complex that consistently breaks apart at the Head/Middle inter-module boundary [30].

\section{Modular organization of the core Mediator complex}

A detailed comparison of the structures of the core Mediator and its subassemblies, combined with nanogold labeling of multiple Mediator subunits of different modules, suggests that the Head + Middle modules form the upper portion of the structure, while the Tail module forms the dense domain at the base (Figure 3D and Supplementary information, Figures S6 and S7). The Head and Middle modules fold back on one another and form an independent triangular structure, which is consistent with previous findings that the Head and Middle modules form a functional core of the Mediator [18, 19]. The position of the Tail module at the base of the core Mediator is consistent with its independent functions in interacting with nucleosomes $[26,27]$ and transcription factors $[2$, 24] (Figure 3D and Supplementary information, Figure S7).

These observations directly challenge previous module segmentations of the core Mediator, in which the Middle/ Tail modules tightly interact with each other and form the upper portion of Mediator, while the Head module is largely separated and corresponds to the dense domain at the base $[7,17]$. The limitations of the previous segmentations were that they were based solely on comparisons of low-resolution EM structures of the core Mediator associated with RNAPII and in free forms [7], which were very ambiguous; furthermore, none of the individual Mediator subunits was localized in the EM structure [7, $8,17]$. Moreover, the theoretical MW suggests that the Tail module should be the largest component and that the 
Head module should be the smallest. The calculated MW of each module in our redefined model is very close to its theoretical MW; however, the previous segmentation model suggests that the MW of each module is roughly the same (Figure 4A).

\section{Functional interaction of Mediator with RNAPII}

Among the Mediator modules, the highly conserved Head module is the most well-characterized, and it interacts directly with the RNAPII machinery [28, 31, 32]. Interestingly, nine Mediator subunits are encoded by Srb genes, which were first identified through a genetic screen for extragenic suppressors of the RNAPII CTD truncation mutations $[33,34]$. Among them, four Srb subunits belong to the Head module and one to the Middle module [21] (Figure 3E). Based on functional and structural studies, the Head module interacts extensively with RNAPII through three putative interaction surfaces on RNAPII: the CTD of the Rpb1 subunit $[13,35]$, the Rpb3 subunit [36] and the Rpb4/7 sub-complex [8, 28]. When characterizing the Mediator and RNAPII interaction in vitro a couple of years ago, RNAPII was typically visualized as binding to the Mediator through the previously defined 'Tail' module, which was far away from the formerly identified 'Head' module with which RNAPII should interact [8]. In contrast, our re-defined model clearly shows that RNAPII binds to the Head module of the core Mediator.

To investigate the interaction modes of MediatorRNAPII, we collected images of Mediator particles incubated with a four-fold molar excess of RNAPII. Two representative interaction modes were identified after the reference-free alignment and classification of the EM images of Mediator interacting with RNAPII (Figure 3E), substantiating that RNAPII binds tightly to the newly defined Head module. After unambiguously fitting the crystal structure of the Head module complexed with RNAPII CTD (PDBID: 4GWQ) [13] into the 3D map of the core Mediator, the orientation of the RNAPII CTD binding to the core Mediator could be directly deduced. The newly identified CTD-binding site on the 'Middle' module that was argued by a recent EM study [20] actually represents the well-established CTD interaction site on the Head module.

The four Srb subunits that belong to the Head module could be deduced from the fitted crystal structure [13], and the only Srb subunit (Med21/Srb7) [29] of the Middle module was clearly localized by nanogold labeling (Figure 3C). Integration of these observations results in the first structural view of the core Mediator-CTD interaction network (Figure 3E), in which the extended CTD interacting extensively with Med17/Srb4 is proximal to the other four Srb subunits of the core Mediator, indicat- ing a much larger interaction surface of the core Mediator for CTD binding. Indeed, it has been suggested that there is an extensive interaction surface on Mediator for CTD binding, which involves multiple Mediator components [3]. In addition to the neck region directly interacting with the extended CTD [13], the joint and moveable jaw regions were also proposed to be involved in CTD binding [14]. Moreover, it is likely that subunits from the other modules also directly interact with CTD because the Head module itself is insufficient for CTD binding [32].

\section{Implications of Mediator's function in the PIC assembly}

In the absence of the Mediator, PIC assembly still occurs in an orderly fashion in vitro [37]. However, the Mediator complex is preeminently required for PIC assembly in vivo, which interacts extensively with RNAPII and general transcription factors. Mediator interacts with RNAPII through CTD of the Rpb1 subunit [13, 35], Rpb3 subunit [36], and Rpb4/7 sub-complex [8, 28]. Moreover, Mediator directly interacts with general transcription factors such as TBP [28, 38], TFIIB [19, 39], and TFIIH [40]. Surprisingly, all such multipartite interactions are dependent on the Head module. Thus, we mapped all of the regions reported to interact with RNAPII and general transcription factors onto the redefined Mediator modular structure and found that the interaction with RNAPII machinery is centered on the jaw and neck regions of the Head module (Figure 4B). An extended network of contacts of the PIC and the Mediator is concentrated on three surfaces: a large portion of the RNAPII surface centered on the docking region, including CTD, Rpb3 and the Rpb4/7 sub-complex; the surfaces of TBP/TFIIB; and the Rad3 subunit of TFIIH. Consistently, according to the most up-to-date structural model of the PIC assembly [37], the RNAPII, TBP/TFIIB and TFIIH surfaces are also exposed and available for functional interactions with the Mediator (Figure 4B). It has been established that TBP/TFIID, TFIIH, TFIIE and Mediator could constitute a scaffold for the formation of a functional reinitiation complex [41], which remains at the promoter after transcriptional initiation. The two potential interaction surfaces of TBP/TFIIB and the Rad3 subunit of TFIIH with the Head module most likely represent a first glimpse at the reinitiation scaffold complex. Taken together, the newly defined modular organization of the core Mediator provides a structural framework for understanding the function of Mediator in PIC assembly.

\section{Discussion}

Since the core Mediator is a low-abundance, fragile and large protein complex, it is extremely difficult to pre- 

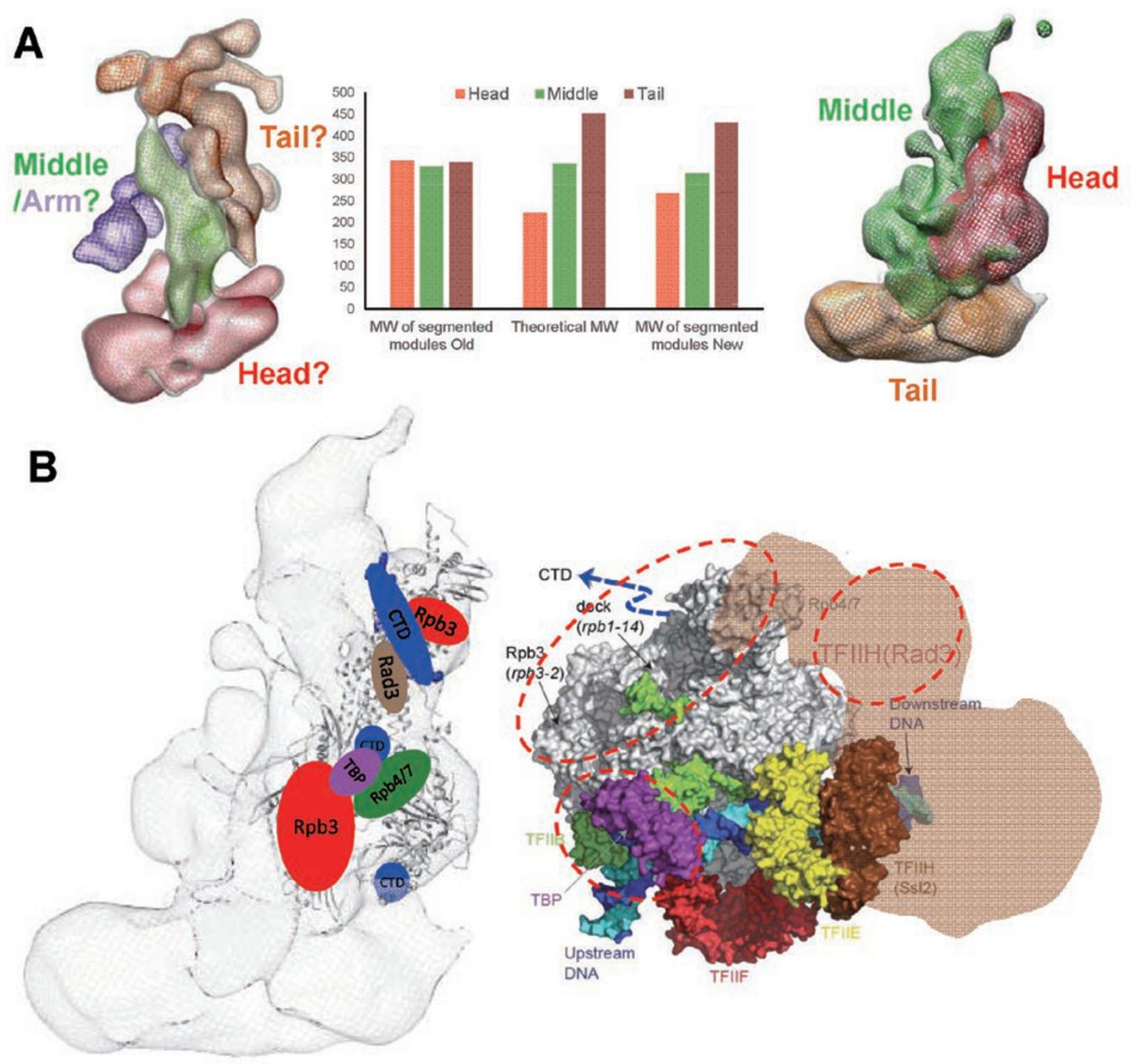

Figure 4 Redefined modular organization of the core Mediator complex and its functional implications. (A) Modular organization of the yeast Mediator complex. Previously defined modular architecture (left) [7, 8, 17], which was solely based on comparing low-resolution EM structures of the core Mediator in RNAPII-associated and free forms. The redefined modular organization (right) is derived from detailed comparison of the structures of the core Mediator and module assemblages, combined with the nanogold labeling of several subunits of different modules. The inserted histogram lists the theoretical MW of each module and the calculated MW obtained from the number of voxels enclosed by each segmented density from both the previous and redefined modular model. (B) Multipartite interactions between Mediator and RNAPII and general transcription factors. The redefined Mediator modular structure fitted with the X-ray structure of the Head module (left, PDBID: 4GWQ) [13] and the surface representation of the RNAPII transcription PIC model (right) [37, 48]. The figure of the PIC model is adapted from a previous study [14], and the position of TFIIH is inferred from structural information on the human PIC [37]. The documented regions of the Mediator Head module that could be responsible for interacting with RNAPII (CTD [13, 35], Rpb3 subunit [36], and Rpb4/7 sub-complex [8, 28]) and general transcription factors (TBP [28, 38] and TFIIH [40]) are labeled with ovals in different colors. The three putative interaction surfaces with Mediator on the PIC are centered on RNAPII (CTD [13, 35], Rpb3 subunit [36], and Rpb4/7 sub-complex [8, 28]), TBP [28, 38], TFIIB [19, 39] and TFIIH [40] (the Rad3 subunit), which are highlighted with red dashed ovals.

pare the specimen that is suitable for structural analyses. Therefore, the basic question about the boundaries of the three modules remained unresolved. The previous mod- ule segmentation was very ambiguous, which was based solely on comparing low-resolution EM structures of the core Mediator in RNAPII-associated and free forms 
[7]. Furthermore, no single core Mediator subunit was localized in the EM structures [8, 17] (Figure 4A). In the previous modular assignment, the Middle + Tail modules interact with each other and form the upper portion of Mediator, and the Head module is separated and corresponds to the dense domain at the base [7, 17]. Unfortunately, this modular assignment apparently contradicts many biochemical and functional studies.

Here we improved the method for isolating the native core Mediator and for the first time determined the 3D structure of the Head + Middle modules, which enabled the precise assignment of all three modules of the core Mediator. The structural comparisons of the core Mediator and the Head + Middle modules permitted the conclusive inference of the Head + Middle and Tail modules. Furthermore, the detailed structural comparison of the Head + Middle and the Head modules contributed to distinguishing the Head and Middle modules. Moreover, we for the first time successfully pinpointed Mediator subunits in the core Mediator by EM. The use of nanogold labeling to precisely localize four Mediator subunits that belong to different modules critically contributed to the modular segmentation of the core Mediator. Our findings directly challenged the previous modular segmentation. According to the redefined modular model, the Head and Middle modules fold back on one another and form the upper portion of the core Mediator, while the Tail module forms a distinct dense domain at the base. The estimated MW of each segmented module in the redefined model also closely matches its theoretical MW (Figure 4A). Collectively, these analyses have completely redefined the modular organization of the core Mediator.

Through the improved biochemical preparation and conformational sorting of the core Mediator particles preserved in negative staining, we acquired the first 3D EM reconstructions of the core Mediator in three different conformations (named collapsed, closed and open). The conformational changes are largely due to the mobility of the Tail module, which could be either far away from or close to the Head + Middle modules. The structure of the Head + Middle module assemblage was largely stable, which is consistent with the Head and Middle modules being the functional core of the Mediator [18, 19]. The high mobility of the Tail module is consistent with the independent capability of the Tail module to interact with transcription factors $[2,24]$ and nucleosomes $[26,27]$. Presumably, the conformational flexibility of the Tail module could integrate the regulation signals from the transcription factors or facilitate the crosstalk between transcriptional regulation and the modulation in chromatin structure.

The previous EM studies suggested that RNAPII binds to the 'Tail' module [8] and that CTD mainly binds to the 'Middle' module of the Mediator [20], which are contradictory to many biochemical and functional studies showing that RNAPII binds to the Head module. After unambiguously redefining the modular organization of the core Mediator, we have, for the first time, reconciled the inconsistency between the structurally and functionally defined Mediator modules. The redefined model is well-compatible with previous functional and biochemical studies. Astonishingly, the entire interaction surface of the Head module on the core Mediator is highly exposed and available for functional interactions with RNAPII and general transcription factors (Figure 4B). It appears that no conformational changes in Mediator are required to expose the interaction surface to achieve the PIC assembly. This observation seems contradictory to a possible mechanism for transcriptional regulation by Mediator [17], whereby a large-scale rearrangement of the Mediator structure was argued to be essential for generating a surface for PIC assembly. Because the Head module alone is insufficient to recapitulate all of the Mediator functions [32], additional contacts between components of the RNAPII basal transcription machinery and other Mediator modules may still be of critical functional significance; for example, they may facilitate the disassembly and re-assembly of the PIC.

Although the Mediator complex is a central controller of eukaryotic transcription, the detailed architecture and molecular mechanisms of the complex remain largely elusive. As the modular architecture and subunit composition of the Mediator are conserved from yeast to human [6], an integrated structural biology approach has been proposed to unravel the detailed architecture and molecular mechanism of Mediator [16]. We have now redefined the modular organization of the core Mediator by EM and integrated the structural and functional information into a coherent mechanism for Mediator modularity and regulation in transcription initiation. This study thus constitutes a critical step toward establishing such an integrated structural biology approach.

\section{Materials and Methods}

\section{Yeast strains}

A modified TAP $(10 \times$ histidine-TEV-ProteinA $)$ tag mTAP [8] was fused to the $\mathrm{C}$ terminus of the Med14, Med17, Med22 or Med21 subunit, using a PCR-based genomic epitope-tagging method in the multiple protease-deficient yeast strain BJ2168 (MATa leu2 trp1 ura3-52 prb1-1122 pep4-3 prc1-407 gal2). To isolate the endogeneous Head + Middle modules, the nonessential Mediator subunit Med16/Sin4 was deleted [23, 24].

Purification of the core Mediator, and the Head + Middle 
modules

Yeast cells were grown in YPD medium. Cells were harvested, washed and re-suspended in extraction buffer $(50 \mathrm{mM}$ HEPES, $\mathrm{pH}$ 7.6, $300 \mathrm{mM}$ KOAc, $0.5 \mathrm{mM}$ EDTA, $5 \mathrm{mM} \beta-\mathrm{ME}, 10 \%$ (v/v) glycerol, $0.1 \%(\mathrm{v} / \mathrm{v}) \mathrm{NP}-40$ and protease inhibitors). For purification of the Head + Middle modules, mTAP tags were inserted to the $\mathrm{C}$ terminus of the Med21 subunits on the background of BJ2168 (Med16A); for preparing the core Mediator complex, mTAP was fused to the C terminus of the Med14, Med17, Med22 or Med21 subunit of BJ2168 strain. First a standard Mediator purification procedure using affinity purification was carried out as previously described [8]. To further improve the purity and homogeneity, the elution from the $\mathrm{IgG}$ column was applied onto a Mono Q column (GE Healthcare) in Q100 buffer (100 mM ammonium sulfate, 50 $\mathrm{mM}$ Tris, $\mathrm{pH}$ 7.6, 10\% v/v glycerol, 1 mM EDTA, $10 \mu \mathrm{M} \mathrm{ZnSO} 4$, $0.02 \% \mathrm{NP}-40,10 \mathrm{mM} \beta-\mathrm{ME}$ ) and was resolved over a 100-1 000 $\mathrm{mM}$ ammonium sulfate gradient. The final fractions of the Head + Middle modules and the core Mediator complex eluted at different ammonium sulfate concentration were flash-frozen in liquid nitrogen. S. pombe Mediator purification was prepared from $S$. pombe strains TP161 [10] as described above. Purified protein complexes were analyzed by SDS-PAGE and examined by EM.

EM sample preparation, data collection and image analysis

About $3 \mu \mathrm{l}$ of aliquots $(\sim 20 \mu \mathrm{g}$ protein $/ \mathrm{ml}$ in $50 \mathrm{mmol} / \mathrm{L}$ Tris, $\mathrm{pH}$ 7.6, $100 \mathrm{mmol} / \mathrm{L}$ ammonium sulfate, $10 \%$ (v/v) glycerol, 1 $\mathrm{mmol} / \mathrm{L}$ EDTA, $10 \mu \mathrm{mol} / \mathrm{L} \mathrm{ZnSO} 4,0.02 \% \mathrm{NP}-40,10 \mathrm{mmol} / \beta-\mathrm{ME})$ was applied to a carbon-coated 400 -mesh $\mathrm{Cu}$ EM specimen grid freshly glow discharged and was then preserved by staining with $0.75 \%(\mathrm{w} / \mathrm{w})$ uranyl formate solution. Images were recorded at a magnification of $62000 \times$ on a $4096 \times 4096 \mathrm{CCD}$ detector (FEI Eagle) with a Tecnai F20 electron microscope (FEI) operating at an acceleration voltage of $200 \mathrm{kV}$. Images were recorded by using low-dose procedures, at $\sim 0.6-0.8 \mu \mathrm{m}$ under focus. Two-fold pixel binning of the original CCD images resulted in a final pixel size of $3.54 \AA$ per pixel. Image processing was carried out with SPIDER [42] and SPARX [43].

The particles of the Head + Middle modules and the core Mediator show a strongly preferred orientation upon adsorption to amorphous carbon support films. To overcome the problems of preferred orientation and substantial structural variability, 3D reconstructions were calculated by using the random conical tilt method [22]. Tilted $\left(-55^{\circ}\right)$ and un-tilted image pairs were obtained under low-dose conditions and particles were selected using the TiltPicker program [44] and montaged them for interactive screening, yielding $\sim 3000$ tilt-pairs images of the Head + Middle modules, and $\sim 5000$ tilt-pairs images of the core Mediator complex. We initially analyzed 2D images using the Relion [45] and Sparx package [43]. We then used the resulting averages to run iterative alternating rounds of supervised multi-reference alignment and classification as well as reference-free alignment with SPIDER [42] to improve the homogeneity of the image classes [46]. We produced all molecular graphics images using Chimera [47].

\section{Nanogold labeling}

Gold cluster labeling of Mediator complex was carried out with purified Mediator samples with 10× Histidine tagged on Med17, Med22, Med14 and Med21 subunits. Incubation of the sample at $20 \mu \mathrm{g}$ protein $/ \mathrm{ml}$ with a 10 -fold molar excess of Ni-NTA (Nanoprobes, Yaphank, NY USA) was carried out for $2 \mathrm{~h}$ at $4{ }^{\circ} \mathrm{C}$, then dialyzed against the buffer (50 mM Tris, $\mathrm{pH} 7.6,100 \mathrm{mM}$ ammonium sulfate, $1 \mathrm{mM}$ EDTA, $10 \mu \mathrm{M} \mathrm{ZnSO} 4$ and $0.01 \% \mathrm{NP}-40$ ) for another $2 \mathrm{~h}$ at $4{ }^{\circ} \mathrm{C}$. EM samples were prepared and imaged as described above.

\section{EM analysis of Mediator-RNAPII interaction}

The complex of Mediator-RNAPII was assembled by incubation Mediator with a four-fold molar excess of RNAPII for $4 \mathrm{~h}$ at $4{ }^{\circ} \mathrm{C}$. The resulting assembly was negatively stained and examined by EM as described above.

\section{Acknowledgments}

This work was supported by the National Basic Research Program (2013CB910200 and 2014CB910700), the National Natural Science Foundation of China (31222017), and the Ministry of Education of PRC (NCET-11-0874). We thank Xuefeng Zhu (University of Gothenburg, Sweden) for providing the TP161 strain for expression of $S$. pombe Mediator.

\section{References}

1 Lee TI, Young RA. Transcription of eukaryotic protein-coding genes. Annu Rev Genet 2000; 34:77-137.

2 Conaway RC, Conaway JW. Function and regulation of the Mediator complex. Curr Opin Genet Dev 2011; 21:225-230.

3 Kornberg RD. Mediator and the mechanism of transcriptional activation. Trends Biochem Sci 2005; 30:235-239.

4 Naar AM, Lemon BD, Tjian R. Transcriptional coactivator complexes. Annu Rev Biochem 2001; 70:475-501.

5 Malik S, Roeder RG. Transcriptional regulation through Mediator-like coactivators in yeast and metazoan cells. Trends Biochem Sci 2000; 25:277-283.

6 Bourbon HM. Comparative genomics supports a deep evolutionary origin for the large, four-module transcriptional mediator complex. Nucleic Acids Res 2008; 36:3993-4008.

7 Asturias FJ, Jiang YW, Myers LC, Gustafsson CM, Kornberg RD. Conserved structures of mediator and RNA polymerase II holoenzyme. Science 1999; 283:985-987.

8 Cai G, Imasaki T, Takagi Y, Asturias FJ. Mediator structural conservation and implications for the regulation mechanism. Structure 2009; 17:559-567.

9 Wang X, Wang J, Ding Z, Ji J, Sun Q, Cai G. Structural flexibility and functional interaction of Mediator Cdk8 module. Protein Cell 2013; 4:911-920.

10 Elmlund H, Baraznenok V, Lindahl M, et al. The cyclindependent kinase 8 module sterically blocks Mediator interactions with RNA polymerase II. Proc Natl Acad Sci USA 2006; 103:15788-15793.

11 Taatjes DJ, Naar AM, Andel F 3rd, Nogales E, Tjian R. Structure, function, and activator-induced conformations of the CRSP coactivator. Science 2002; 295:1058-1062.

12 Naar AM, Taatjes DJ, Zhai W, Nogales E, Tjian R. Human CRSP interacts with RNA polymerase II CTD and adopts a specific CTD-bound conformation. Genes Dev 2002; 16:13391344.

13 Robinson PJ, Bushnell DA, Trnka MJ, Burlingame AL, Kornberg RD. Structure of the mediator head module bound to the carboxy-terminal domain of RNA polymerase II. Proc Natl 
Acad Sci USA 2012; 109:17931-17935.

14 Lariviere L, Plaschka C, Seizl M, Wenzeck L, Kurth F, Cramer P. Structure of the Mediator head module. Nature 2012; 492:448-451.

15 Imasaki T, Calero G, Cai G, et al. Architecture of the Mediator head module. Nature 2011; 475:240-243.

16 Lariviere L, Seizl M, Cramer P. A structural perspective on Mediator function. Curr Opin Cell Biol 2012; 24:305-313.

17 Chadick JZ, Asturias FJ. Structure of eukaryotic Mediator complexes. Trends Biochem Sci 2005; 30:264-271.

18 Liu Y, Ranish JA, Aebersold R, Hahn S. Yeast nuclear extract contains two major forms of RNA polymerase II mediator complexes. J Bio Chem 2001; 276:7169-7175.

19 Kang JS, Kim SH, Hwang MS, Han SJ, Lee YC, Kim YJ. The structural and functional organization of the yeast mediator complex. J Bio Chem 2001; 276:42003-42010.

20 Tsai KL, Sato S, Tomomori-Sato C, Conaway RC, Conaway JW, Asturias FJ. A conserved Mediator-CDK8 kinase module association regulates Mediator-RNA polymerase II interaction. Nat Struct Mol Biol 2013; 20:611-619.

21 Guglielmi B, van Berkum NL, Klapholz B, et al. A high resolution protein interaction map of the yeast Mediator complex. Nucleic Acids Res 2004; 32:5379-5391.

22 Radermacher M. Three-dimensional reconstruction of single particles from random and nonrandom tilt series. $J$ Electron Microsc Tech 1988; 9:359-394.

23 Li Y, Bjorklund S, Jiang YW, et al. Yeast global transcriptional regulators Sin4 and Rgr1 are components of mediator complex/RNA polymerase II holoenzyme. Proc Natl Acad Sci USA 1995; 92:10864-10868.

24 Zhang F, Sumibcay L, Hinnebusch AG, Swanson MJ. A triad of subunits from the Gal11/tail domain of Srb mediator is an in vivo target of transcriptional activator Gen $4 \mathrm{p}$. Mol Cell Biol 2004; 24:6871-6886.

25 Baidoobonso SM, Guidi BW, Myers LC. Med19 (Rox3) regulates Intermodule interactions in the Saccharomyces cerevisiae mediator complex. J Bio Chem 2007; 282:5551-5559.

26 Zhu X, Zhang Y, Bjornsdottir G, et al. Histone modifications influence mediator interactions with chromatin. Nucleic Acids Res 2011; 39:8342-8354.

27 Lorch Y, Beve J, Gustafsson CM, Myers LC, Kornberg RD. Mediator-nucleosome interaction. Mol Cell 2000; 6:197-201.

28 Cai G, Imasaki T, Yamada K, Cardelli F, Takagi Y, Asturias FJ. Mediator head module structure and functional interactions. Nat Struct Mol Biol 2010; 17:273-279.

29 Baumli S, Hoeppner S, Cramer P. A conserved mediator hinge revealed in the structure of the MED7.MED21 (Med7.Srb7) heterodimer. J Bio Chem 2005; 280:18171-18178.

30 Linder T, Zhu X, Baraznenok V, Gustafsson CM. The classical srb4-138 mutant allele causes dissociation of yeast Mediator. Biochem Biophys Res Commun 2006; 349:948-953.

31 Cai G, Chaban YL, Imasaki T, et al. Interaction of the Mediator head module with RNA polymerase II. Structure 2012; 20:899-910.

32 Takagi Y, Calero G, Komori H, et al. Head module control of mediator interactions. Mol Cell 2006; 23:355-364.

33 Nonet ML, Young RA. Intragenic and extragenic suppressors of mutations in the heptapeptide repeat domain of Saccharomyces cerevisiae RNA polymerase II. Genetics 1989; 123:715-724.
34 Thompson CM, Koleske AJ, Chao DM, Young RA. A multisubunit complex associated with the RNA polymerase II CTD and TATA-binding protein in yeast. Cell 1993; 73:1361-1375.

35 Thompson CM, Young RA. General requirement for RNA polymerase II holoenzymes in vivo. Proc Natl Acad Sci USA 1995; 92:4587-4590.

36 Soutourina J, Wydau S, Ambroise Y, Boschiero C, Werner M. Direct interaction of RNA polymerase II and mediator required for transcription in vivo. Science 2011; 331:1451-1454.

37 He Y, Fang J, Taatjes DJ, Nogales E. Structural visualization of key steps in human transcription initiation. Nature 2013; 495:481-486.

38 Lariviere L, Geiger S, Hoeppner S, Rother S, Strasser K, Cramer P. Structure and TBP binding of the Mediator head subcomplex Med8-Med18-Med20. Nat Struct Mol Biol 2006; 13:895-901.

39 Baek HJ, Kang YK, Roeder RG. Human Mediator enhances basal transcription by facilitating recruitment of transcription factor IIB during preinitiation complex assembly. J Bio Chem 2006; 281:15172-15181.

40 Esnault C, Ghavi-Helm Y, Brun S, et al. Mediator-dependent recruitment of TFIIH modules in preinitiation complex. Mol Cell 2008; 31:337-346.

41 Yudkovsky N, Ranish JA, Hahn S. A transcription reinitiation intermediate that is stabilized by activator. Nature 2000; 408:225-229.

42 Frank J, Radermacher M, Penczek P, et al. SPIDER and WEB: processing and visualization of images in 3D electron microscopy and related fields. J Struct Biol 1996; 116:190199.

43 Hohn M, Tang G, Goodyear G, et al. SPARX, a new environment for Cryo-EM image processing. J Struct Biol 2007; 157:47-55.

44 Voss NR, Yoshioka CK, Radermacher M, Potter CS, Carragher B. DoG Picker and TiltPicker: software tools to facilitate particle selection in single particle electron microscopy. $J$ Struct Biol 2009; 166:205-213.

45 Scheres SH. RELION: implementation of a Bayesian approach to cryo-EM structure determination. J Struct Biol 2012; 180:519-530.

46 Brignole EJ, Smith S, Asturias FJ. Conformational flexibility of metazoan fatty acid synthase enables catalysis. Nat Struct Mol Biol 2009; 16:190-197.

47 Pettersen EF, Goddard TD, Huang CC, et al. UCSF Chimera-a visualization system for exploratory research and analysis. J Comput Chem 2004; 25:1605-1612.

48 Grunberg S, Warfield L, Hahn S. Architecture of the RNA polymerase II preinitiation complex and mechanism of ATPdependent promoter opening. Nat Struct Mol Biol 2012; 19:788-796.

(Supplementary information is linked to the online version of the paper on the Cell Research website.)

(c) (i) () $(-)$ This work is licensed under the Creative Commons Attribution-NonCommercial-No Derivative Works 3.0 Unported License. To view a copy of this license, visit http:// creativecommons.org/licenses/by-nc-nd/3.0 